\title{
Middleware for Multi-Interfaces Management Through Profiles Handling
}

\author{
Jean-Marie Bonnin, Zied Ben Hamouda, \\ Imed Lassoued \\ Telecom Bretagne \\ 35576, Cesson Sévigné, France \\ \{jm.bonnin, mouhamed.benhamouda, \\ imed.lassoued\}@enst-bretagne.fr
}

\author{
Addelfattah Belghith \\ Ecole Nationale de Sciences de l'Informatique \\ Campus de la Manouba \\ 2010 Manouba, Tunisia \\ abdelfattah.belghith@ensi.rnu.tn
}

\begin{abstract}
Mobile wireless technologies have seen an immense growth in the recent years. Different kink of wireless public access have become increasingly available in areas like airports, stations and shopping centers as well as at office or at home. Mobile terminal such as phone or laptop are often equipped with several network interfaces and may use several access networks such as UMTS, WiMAX, WiFi and BlueTooth; each of them can be attached to different Internet providers having different billing models and security levels. To allow mobile users to be always connected to the best available access network and maintain theirs connections when they switch from one network to another, several vertical handoff mechanisms have been recently proposed. Few of them deal properly with the cost in the decision process, which should be done at a per flow basis. Our current work focuses on an advanced middleware that allows, through a comprehensive profile management, the support of automatic interfaces configuration and per-flow interface selection taking into account preferences given by the terminal's owner and applications. As illustrative, we show how the proposed middleware allows cost effective management of a multi-interfaces terminals fleet through an easy to configure set of preferences and resources profiles.
\end{abstract}

\section{Categories and Subject Descriptors}

C.2.1 [Computer-Communication Networks]: Network Architecture and Design-Wireless Communication

\section{Keywords}

Vertical Handover Middleware, Profile Management

\section{INTRODUCTION}

Many companies thrive today to manage more rigorously their mobile terminal fleets, where mobile terminals might be connected through different communication technologies

Permission to make digital or hard copies of all or part of this work for personal or classroom use is granted without fee provided that copies are not made or distributed for profit or commercial advantage and that copies bear this notice and the full citation on the first page. To copy otherwise, to republish, to post on servers or to redistribute to lists, requires prior specific permission and/or a fee. MOBILWARE 2008, February 13-15, Innsbruck, Austria

Copyright @ 2008 ICST 978-1-59593-984-5

DOI 10.4108/ICST.MOBILWARE2008.2899 provided by several operators. Their main goal is to efficiently maintain operational services supporting employees mobility, and to control their expenses and energetic costs. Recent years have witnessed a progressive adoption of wireless technologies as they allow companies to increase their productivity. Nonetheless, the use of these technologies has a direct impact on the company operating budget. This impact is particularly due to the recent introduction of the $3 \mathrm{G}$ technologies, the proliferation of WiFi hotspots, the popularity of text (SMS) and multimedia messaging (MMS) that enable users to relay images, video clips and MP3 files among others, yet increase the company expense.

At the same time, the integration of various heterogeneous access networks in a ubiquitous wireless environment is on the way (see e.g., [1], [2] and [14]). Researches are focusing on creating an unified platform architecture providing an ubiquitous integration among heterogeneous technologies. Each technology presents a certain bandwidth and works at a given coverage. Keeping the mobile user always connected while using for each running applications the adequate technology among the current available technologies while providing the best tradeoff between bandwidth, bit error rate, one way delay and its induced cost, is an essential requirement. With the integration of access technologies, the interface selection problem for a multi-interfaces mobile terminal has gained importance in recent years (e.g., [8], [17], [3]). Various recent works have proposed vertical handoff solutions where mobile users can move among various network types (see e.g., [15] and [9]). In these works, two basic goals have already and clearly emerged. The first one states that users should be provided with seamless roaming amongst various access networks (e.g. [10]), including simultaneous or successive connections through several access technologies. The second one mandates that users should be allowed to always stay connected through the "best" access network (e.g. [6], [7]).

Nonetheless, to the best of our knowledge, the majority of these vertical handoff proposals integrate an optimal interface selection process but do not provide any mechanism allowing a comprehensive terminals connectivity management. The use of such proposals does not allow to control some essential parameters such as monetary costs, device power consumption and service satisfaction.

In this paper, we show how a middleware, named Ubique, allows to control the behavior of multi-interfaces terminals using an easy to manage set of profiles. These profiles are used with actual statistics to feed-up the interface selection 
mechanism. The rest of the paper is organized as follows. After the presentation of related research works, we briefly review the Ubique architecture and its functionalities. Then, we introduce the profiling mechanism upon which our middleware is based. In order to show how Ubique fits the need of fleet management, we detail one sort of profiles, namely the Preferences and Resources Profile (PRP) and describe the validation scenario used to highlight the capability of our middleware to manage a fleet of multi-interfaces mobile terminals.

\section{RELATED WORKS}

Currently, there are a growing number of research and standardization efforts related to user management through the definition and the handling of several profiles. For example, in [11], the notion of a Generic User Profile (GUP) is defined as being a collection of data stored and managed by different entities such as the user equipment, the home or visited networks, and which affects the way the end-user experiences the different services offered. The WAP User Agent Profile (UAProf), defined in [12], is concerned with capturing classes of device capabilities and preferences. The Composite Capability/Preference Profiles (CC/PP) framework (see [13]) is yet another mechanism for handling the preferences associated to users and user agents accessing the World Wide Web. The Information Society Technologies (IST) Aquila project tries to provide dynamic control to DiffServ based traffic and defines application profiles which contain the concrete application's needs description. Moreover, the IST-TRUST project tries to understand the user requirements related to reconfigurable radio systems. It also defines a layered architecture which contains a component for policies and profiles management. These profiles are further refined within the IST-SCOUT project.

Two main approaches have been considered for handover decision making in heterogeneous access networks: the network-controlled (NCHO) and mobile-assisted handover (MA$\mathrm{HO})$ and the mobile-controlled handover (MCHO). In the first one, the handover is triggered and executed by the network taking into account contextual information given by the mobile. The architecture proposed in [22], for instance, defines a Vertical Handoff Decision Controller (VHDC) which collects informations about heterogeneous network and users mobility. In the MCHO approach, all decisions are made in the mobile node [19]. Anyway, it can rely on information provided by the network such as the list of available radio access networks [18].

In [21] authors present a terminal-oriented approach called universal seamless handoff architecture (USHA) which use a handoff server to communicate with mobile users through tunnels. In [20] authors present a mobility management approach controlled by the terminal which have a clear view of the current context to guarantee seamless mobility between heterogeneous networks. Following these trends, we have recently developed a middleware that integrates management mechanisms for user profiling to support vertical handovers over various access technologies. Our middleware named Ubique allows mobile terminals to automatically select the best interface for each application flow while taking into account various requirements, such as user/administrator preferences, the surrounding context and the application needs. Furthermore, Ubique allows the management of mobile terminal through a comprehensive set of profiles. Among them,
Preferences and Resources Profiles (PRP) specify the administrator, users and applications preferences.

\section{THE $U B I Q U E$ ARCHITECTURE}

In order to allow users to take advantages of the simultaneous availability of different access networks, an advanced middleware, named Ubique, was designed and implemented. This middleware allows a multi-interfaces mobile terminal, having various connections to several access technologies and serving different applications, to automatically configure and select the best suitable set of interfaces according to a comprehensive set of preferences.

To achieve these goals, Ubique integrates a user profiling management mechanism with an optimized interface selection process [3]. It investigates different factors such as interface capabilities, access network characteristics, application requirements and user/administrator preferences. All necessary information is specified in profiles and the decision to use (i.e. activate) an interface and to map a flow on it take them into account. Fig. 1 shows the Ubique framework and its interactions with external entities.

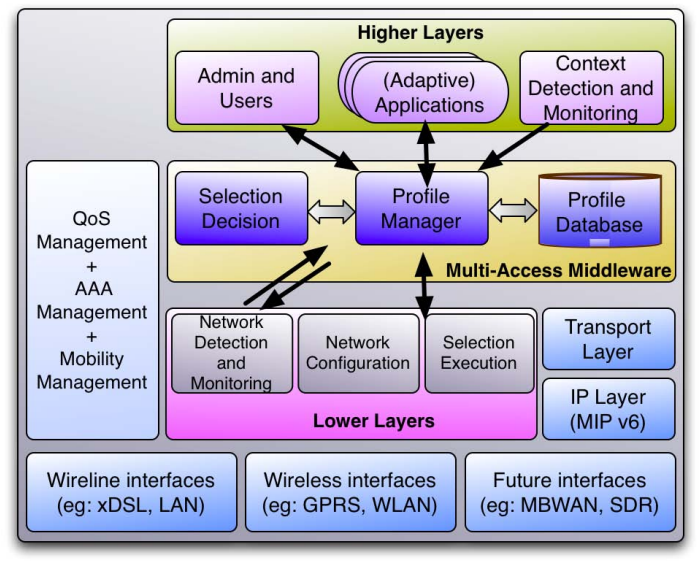

Figure 1: Ubique architecture

Ubique can be seen as a middleware between two system layers: "Higher-Layers" and "Lower-Layers" as portrayed in Fig. 1. The "Higher-Layers" provide the middleware with the User/Administrator preferences, the applications requirements and the system information defining the current context in which the interface selection decision has to be made (e.g., battery level state, terminal GPS locations, time of the day, etc). The "Low- Layers" are rather composed of three different modules. The Network Detection and Monitoring detects available access networks and gives real-time information about interfaces and access network capabilities. The Network Configuration module, performs on demand network interface activations and configurations in order to use the underlying access networks. The the Selection Execution, maps application flows to the "best-suited" interfaces.

The middleware itself has three main components: the Profile Manager (PM), the Selection Decision Algorithm (SDA) and the Profile Database (PDB) (see fig 1). A preliminary simplified version of this architecture have been developed [3]. It uses an integrated design that required to modify IP and MAC layers in order to support communica- 
tion on multiple interfaces simultaneously. The new design benefits from latest development of the Mobile IP architecture and uses the MCoA (Multiple Care-of Addresses) capabilities to support multiple interfaces [23]. This avoid to have to perform a lot of specific modifications in the host system.

\subsection{The Profile Manager (PM)}

This is the main component of the middleware. It receives triggers from external components (i.e., system, applications) and performs all required operations among the internal components. These operations may finally result in other triggers which are sent by the PM to external components. Hence, the PM acts as a coordinator within the architecture. It interacts with all entities which supply the information gathered in profiles it manages. It determines whether the SDA should be launched to make a new decision.

\subsection{The Selection Decision Algorithm (SDA)}

Using a method based on a Multi-Objective optimization [16], this component decides which are the best interfaces to use taking into account internal and external environment. The decision here is make on a flow-per-flow basis according to the information stored in the PDB. The SDA does not need either to know how the parameters are collected within the various profiles or how the selection decisions are enforced. It is worth to note that the SDA could implement diverse selection decision process, but few of those described in the literature (see [24]) are able to take into account such a large panel of parameters as well as to work on a flow-perflow basis.

\subsection{The Profile Data Base (PDB)}

This module stores the various existing profiles and summarizes information about components of the system and their interactions with the environment. More specifically, the profile mechanism serves the following purposes:

- Automating the selection of an interface and its configuration within an access network by maintaining all the necessary information in the PDB.

- Assisting the SDA while it decides for the "best" access option.

- Informing the adaptive applications about actual capabilities of available interfaces and access networks.

Four types of profile have been defined; they may be augmented by the system administrator if necessary. Theses generic profiles are described as generic schemas which specify the structure, the different parameters and their default values. They can be seen as patterns and implemented using XML schemas.

- Preferences and Resources Profile (PRP): This profile defines the administrator, the user and application preferences. This includes, for example, the security level required and preferred and forbidden access networks. It has been generally observed that one's preferences usually depend on the currently existing resources, the current traffic and connection requirements and the surrounding in which the mobile terminal is operating. Thus, the PRP provides specifications on how the system should behave for several contexts (e.g., battery status, time of the day, GPS location, screen size, etc).

- Flow Description Profile (FDP): The specifies the QoS parameters an application requiers for one of its flow. It comprises two parts: the applications QoS requirements (e.g., service class, minimum necessary bit rate, typical delay expected, maximum delay variation) and the QoS monitored by the system (e.g., mean bit rate, bit error rate, average latency). Default FDP may exist for common applications and especially for non Ubique-aware ones.

- Network Interface Profile (NIP): It defines network interface related parameters. Usually this kind of information can be obtained from technical specifications (e.g., maximum theoretical throughput supported) or through measurements (e.g., maximum real throughput). Apart from this static component, the NIP may also contain statistics obtained with the help of the network monitoring entity (e.g. average bit rate, monitored error rate).

- Access Network Profile (ANP): This profile specifies the Layer 2 and Layer 3 information needed to successfully configure and use a network interface with a specific access network such as WEP keys for IEEE $802.11 \mathrm{a} / \mathrm{b} / \mathrm{g}$ hotspots, PIN codes for Bluetooth, an access point name for GPRS networks. This profile also defines the monetary cost and the security level associated with an access network. One part of this profile is static (e.g., network name) and the other part is dynamic (for example, the actual cost associated to the communication).

Generic profiles are instantiated in specific profiles when they are filled either by the administrator or by the users. For instance, when a new physical network interface is added to the computer, a new NIP is created. It contains all the informations the middle-ware needs to handle this kind of interfaces. This NIP profile can be provided by the manufacturer of the network interface. Then, one or more ANP specific profiles are created to gather all configuration parameters necessary to attach this interfaces to a specific operator. This kind of profile could be provided by the user or directly by the network operator. Based on these profiles the system can configure the new network interface and the SDA knows what performances can be expected from it. The case of PRP profiles which deal with administrator's, user's and application's preferences is managed differently and it will be detailled further in the next section.

Profiles become active when they are currently in use by the selection process. This can happen when a network interface is plugged (NIP) in the system, when an access network becomes available (ANP) or when all preferences are gathered $(\mathrm{PRP})$ through a filtering mechanism in a unique active preference profile (see next section).

\section{PRP AND ITS HANDLING MECHANISM}

The SDA can handle only one set of preferences, this active PRP is obtained by "filtering" (in Computer Science acceptation) all relevant PRPs (the "relevance" depend on the context (e.g. battery status)). In fact, as the administrator 
(or network operators), the users and the applications may have their own specific PRP, we need to filter somehow the various values defined for the same parameter. The filtering mechanism is managed by the following rules:

- All parameters have a value type, which can be mandatory or proposed. A mandatory value is always predominant.

- The priority for a proposed parameter value increases from administrator to user, then to application.

- If there are mandatory parameter values, their priority decreases from administrator to user, then to application.

- Some parameters may obey to a mutual exclusion rule, e.g., if the administrator sets a forbidden network for a user, the user can set the same access network to "preferred", but its preference is ignored in this case.

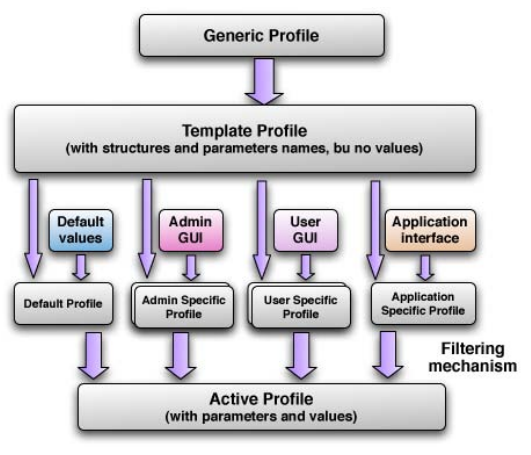

Figure 2: Different types of profiles

\subsection{Preferences and Resources Profile (PRP)}

Recall that the Preferences and Resources Profile (PRP) specifies the way the system should behave based on the available resources and the current situation in which the mobile terminal is operating. The system itself provides the information about network resources currently available (i.e., the current context). Other processes or a combination of processes can provide information used to choose the section of the PRP to be used. This way usage preferences may depend on the current battery status, the time of the day, the GPS location, the screen size or whatever the system need to take into account. Many preference parameters can be specified within this profile, examples of these parameters are:

- Forbidden networks: This parameter prevents the user (i.e. all applications launched by that user) or just a particular application to communicate through these access networks. This parameter can be provided by the administrator and the users.

- Preferred access networks within the same technology: This parameter specifies the preferred GPRS network(s) amongst all the GPRS access networks available to the mobile the terminal. The administrator and/or the user can provide values for this parameter.
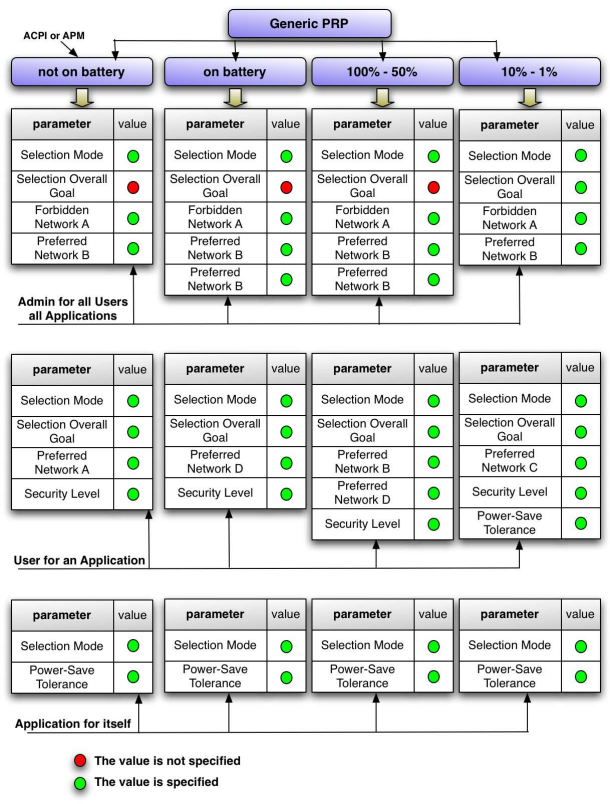

Figure 3: Preferences and resources profiles

The administrator may take into account the different service agreements established with different operators, and the user may take into account the quality of the service to be provided based on his own evaluation and experience.

- Selection overall goal: This parameter indicates which goal is more important when selecting the best interfaces, the monetary cost, the energy consumption or the Quality of Service.

- Application's security level: This parameter defines the security preferences for an application. The administrator, the user and the "Ubique-aware" applications can provide values for this parameter.

- Applications power-save tolerance: Power-save mechanisms impose some constraints on traffic flows and QoS metrics. The application power-save tolerance specifies whether the application can still run properly through a network interface using the powersave mode. The administrator, the user and the "Ubique-aware" applications can provide values for this parameter. The administrator and the user can provide this parameter for a whole class of applications (e.g. for streaming applications).

- Association probe frequency for an access network: This parameter indicates how often to probe an access network (usually a wireless one) in order to get the network interface ready for communication. The administrator and the user can provide values for this parameter.

The administrator can specify a global PRP for all users and all applications, a PRP for all users and a particular application, and a PRP for all applications and a particular user (see Fig. 3). Furthermore, the administrator can 
define a specific PRP just for a particular user and a particular application. The end-user can also specify a PRP for all applications that she/he could launch, or she/he can define a PRP only for a particular application. Of course, an application can define a PRP for itself only, this could be done during the installation.

\subsection{Filtering PRPs}

The active PRP is obtained applying a specific filtering process. Since a large number of PRPs may be involved, we propose to execute the filtering process in two stages (a 2-pass filtering process as portrayed in Fig. 4). In the first pass, we separately filter the administrator specific PRPs and the user specific PRPs. Next, we execute the second pass and we chose as final value the last proposed parameter value or the first mandatory parameter value found in the last column according to the defined filtering process.

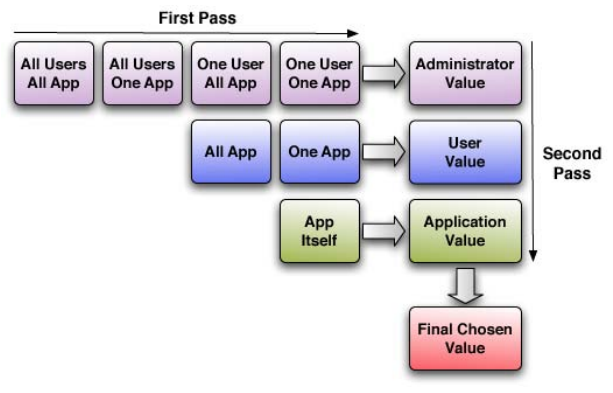

Figure 4: Obtaining an Active PRP

\section{PRP IN FLEET MANAGEMENT}

Recall that PRP profiles contain the administrator, the user or application preferences. Preferences may be related to the security level required by the system or a specific application as well as the preferred and forbidden access networks. The integration of these profiles within our middleware selection decision mechanism allows the management of mobile terminal fleets by controlling several parameters such as the monetary costs and the energy consumption.

In this section, we describe one scenario that we have simulated to study the interest of PRP management mechanism integrated in Ubique for fleet management. In this scenario, we compare Ubique middleware with a mechanism which does not integrate a PRP-like mechanism within the selection decision process.

\subsection{Scenario description}

We performed several test-case simulations to study the PRP interests. In this paragraph we present one of this scenarios. The idea is to simulate the working day of a group of a company's technicians. We assume in this scenario that each technician have a multi-interfaces terminal with IEEE 802.11b, GPRS and Bluetooth network interfaces.

The technicians, move in a city and among customers to perform breakdown services. On the ways, in the offices buildings and in some others areas like shopping centers, stations and coffees there are IEEE $802.11 \mathrm{~b}$ hotspots networks. Some places of the company and its partner's buildings are covered with Bluetooth. Finally, we assume that the GPRS provides coverage every where. Table 1 summa- rizes the characteristics of each access network introduced in our scenario.

\begin{tabular}{|c|c|c|c|c|c|c|}
\hline $\begin{array}{c}\text { Net } \\
\text { ID }\end{array}$ & $\begin{array}{c}\text { Network } \\
\text { Name }\end{array}$ & $\begin{array}{c}\text { Tech- } \\
\text { nology }\end{array}$ & Location & Security & $\begin{array}{c}\text { User } \\
\text { monetary } \\
\text { cost }\end{array}$ & $\begin{array}{c}\text { Company } \\
\text { monetary } \\
\text { cost }\end{array}$ \\
\hline 1 & $\begin{array}{c}\text { Wi } \downarrow- \\
\text { Home }\end{array}$ & $\begin{array}{c}\text { IEEE- } \\
802.11 \mathrm{~b}\end{array}$ & Home & Yes & Free & Duration \\
\hline 2 & $\begin{array}{c}\text { Wi } \downarrow- \\
\text { Work }\end{array}$ & $\begin{array}{c}\text { IEEE- } \\
802.11 \mathrm{~b}\end{array}$ & Work & Yes & Free & Duration \\
\hline 3 & $\begin{array}{c}\text { Work- } \\
\text { Hotspot }\end{array}$ & $\begin{array}{c}\text { IEEE- } \\
802.11 \mathrm{~b}\end{array}$ & Streets & No & Free & Duration \\
\hline 4 & $\begin{array}{c}\text { Private- } \\
\text { Hotspot }\end{array}$ & $\begin{array}{c}\text { IEEE- } \\
802.11 \mathrm{~b}\end{array}$ & Streets & No & Duration & - \\
\hline 5 & $\begin{array}{c}\text { Public- } \\
\text { Hotspot }\end{array}$ & $\begin{array}{c}\text { IEEE- } \\
802.11 \mathrm{~b}\end{array}$ & Stations & No & Free & Free \\
\hline 6 & $\begin{array}{c}\text { Blue- } \\
\text { tooth }\end{array}$ & $\begin{array}{c}\text { Blue- } \\
\text { tooth }\end{array}$ & Work & Yes & Free & Free \\
\hline 7 & GPRS & GPRS & Every- & Yes & Free & Cost/byte \\
\hline
\end{tabular}

Table 1: Networks Description

We assume that each technician can launch the applications listed in Table 2, in which "Professional" application type denotes the applications used by employees acheiving their works, and "Personal" type, applications which are devoted to leisure. Applications flows characteristics came from [5] and [4].

\begin{tabular}{|c|c|c|c|c|c|}
\hline $\begin{array}{c}\text { Application } \\
\text { ID }\end{array}$ & Type & $\begin{array}{c}\text { Flows } \\
\text { Classes }\end{array}$ & $\begin{array}{c}\text { Necessary } \\
\text { Bit-Rate } \\
(\mathbf{k b p s})\end{array}$ & BER & $\begin{array}{c}\text { One-Way } \\
\text { Delay } \\
(\mathbf{m s})\end{array}$ \\
\hline VoIP-Pro & Professional & Conversational & 20 & $10^{-3}$ & 100 \\
\hline VoIP-Perso & Personal & Conversational & 20 & $10^{-3}$ & 100 \\
\hline Visio-Conf- & Professional & Streaming & 64 & $10^{-5}$ & 100 \\
Pro & & Conversational & 20 & $10^{-3}$ & 100 \\
\hline Visio-Conf- & Personal & Streaming & 64 & $10^{-5}$ & 100 \\
Perso & & Conversational & 20 & $10^{-3}$ & 100 \\
\hline E-Mail- & Professional & Background & 1 & $10^{-9}$ & TCP \\
Pro & & & 1 & $10^{-9}$ & TCP \\
\hline E-Mail- & Personal & Background & & & Timer \\
\hline Perso & & & 1 & $10^{-8}$ & 1000 \\
\hline SSH & Professional & Interactive & 1 & 1000 \\
\hline I-Chat & Personal & Interactive & 1 & $10^{-8}$ & \\
\hline
\end{tabular}

Table 2: Scenario applications

We assume finally that two PRP have been define, the administrator (table 3) and the user PRP (table 4). In these PRPs, PlugIn, Full and Low correspond to terminal battery states. PN and FN denote the lists of preferred and forbidden access networks for each application (an access network is identified by its ID).

For example, in Table 3, the administrator specified in the first line that when the battery state is "PlugIn" or "Full", the preferred access networks for the application "VoIP-Pro" are "Wif-Home", "Wif-Work", "Work-Hotspot", "Public-Hotspot", "Bluetooth" and "GPRS"; likewise, there is no forbidden networks for this application. He/She also specified that "VoIPPro" must not be launched through the GPRS access networks when the battery state is "Low". Users specified, as it is shown in Table 4, that the "Private-Hotspot" access net- 
work is always forbidden for all professional applications.

\begin{tabular}{|c|c|c|c|}
\hline $\begin{array}{l}\text { Application } \\
\text { ID }\end{array}$ & Plug-In & Full & Low \\
\hline VoIP-Pro & 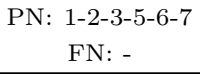 & 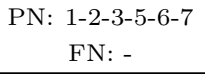 & $\begin{array}{c}\mathrm{PN}: 1-2-3-5-6 \\
\text { FN: } 7\end{array}$ \\
\hline VoIP-Perso & $\begin{array}{c}\text { PN: }- \\
\text { FN: } 1-2-3-6-7\end{array}$ & $\begin{array}{c}\text { PN: }- \\
\text { FN: } 1-2-3-6-7\end{array}$ & $\begin{array}{c}\mathrm{PN}:- \\
\mathrm{FN}: 1-2-3-4-5-6-7\end{array}$ \\
\hline $\begin{array}{c}\text { Visio-Conf- } \\
\text { Pro }\end{array}$ & 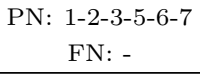 & 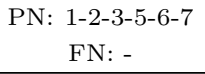 & $\begin{array}{c}\mathrm{PN}: 1-2-3-5-6 \\
\text { FN: } 7\end{array}$ \\
\hline $\begin{array}{c}\text { Visio-Conf- } \\
\text { Pro }\end{array}$ & $\begin{array}{c}\text { PN: }- \\
\text { FN: } 1-2-3-6-7\end{array}$ & $\begin{array}{c}\text { PN: }- \\
\text { FN: } 1-2-3-6-7\end{array}$ & $\begin{array}{c}\text { PN: }- \\
\text { FN: } 1-2-3-4-5-6-7\end{array}$ \\
\hline E-Mail-Pro & $\begin{array}{c}\text { PN: } 1-2-5-6 \\
\text { FN: } 3-7\end{array}$ & $\begin{array}{c}\text { PN: } 1-2-5-6 \\
\text { FN: } 3-7\end{array}$ & $\begin{aligned} & \text { PN: } 6 \\
\text { FN: } & 1-2-3-4-5-7\end{aligned}$ \\
\hline $\begin{array}{c}\text { E-Mail- } \\
\text { Perso }\end{array}$ & $\begin{array}{c}\text { PN: } 1-2-5-6 \\
\text { FN: } 3-7\end{array}$ & $\begin{array}{c}\text { PN: } 1-2-5-6 \\
\text { FN: } 3-7\end{array}$ & $\begin{aligned} \text { PN: } 6 \\
\text { FN: } 1-2-3-4-5-7\end{aligned}$ \\
\hline $\mathrm{SSH}$ & $\begin{array}{c}\text { PN: } 1-2-5-6 \\
\text { FN: } 3-7\end{array}$ & $\begin{array}{c}\text { PN: } 1-2-5-6 \\
\text { FN:3-7 }\end{array}$ & $\begin{array}{c}\text { PN: } 6 \\
\text { FN: } 1-2-3-4-5-7\end{array}$ \\
\hline I-Chat & $\begin{array}{c}\text { PN: }- \\
\text { FN: } 1-2-3-6-7\end{array}$ & $\begin{array}{c}\text { PN: - } \\
\text { FN: } 1-2-3-6-7\end{array}$ & $\begin{array}{c}\text { PN: }- \\
\text { FN: } 1-2-3-4-5-6-7\end{array}$ \\
\hline
\end{tabular}

Table 3: Fleet administrator PRP configuration

Note that the aim of this scenario is to prove the impact of the PRP integration and configuration on the management of a fleet of multi-interfaces terminals; therefore, we measured metrics which show our middleware ability to manage a fleet in a cost and energy effective way, namely monetary costs and power consumption. Likewise, we also evaluate the impact of the PRP management on applications requirements satisfaction measuring the flows availability time and the mean throughput per flow.

\begin{tabular}{|c|c|c|c|}
\hline $\begin{array}{c}\text { Application } \\
\text { ID }\end{array}$ & Plug-In & Full & Low \\
\hline VoIP-Pro & PN: - & PN: - & PN: - \\
& FN: 4 & FN: 4 & FN: 4-7 \\
\hline VoIP-Perso & PN: 1-2 & PN: 1-2 & PN: 1-2 \\
& FN: - & FN: - & FN: 7 \\
\hline Visio-Conf- & PN: - & PN: - & PN: - \\
Pro & FN: 4 & FN: 4 & FN: 4-7 \\
\hline Visio-Conf- & PN: 1-2 & PN: 1-2 & PN: 1-2 \\
Perso & FN: - & FN: - & FN: 7 \\
\hline E-Mail-Pro & PN: - & PN: - & PN: - \\
& FN: 4-7 & FN: 4-7 & FN: 1-2-3-4-5-7 \\
\hline E-Mail-Perso & PN: - & PN: - & PN: - \\
& FN: 4-7 & FN: 4-7 & FN: 1-2-3-4-5-7 \\
\hline SSH & PN: - & PN: - & PN: - \\
& FN: 4-7 & FN:4-7 & FN: 1-2-3-4-5-7 \\
\hline I-Chat & PN: - & PN: - & PN: - \\
& FN: 4-7 & FN: 4-7 & FN: 1-2-3-4-5-7 \\
\hline
\end{tabular}

Table 4: User PRP configuration

\subsection{Simulation Setup}

An emulator has been built to study multi-access middlewares, and especially (i.e., Ubique). We use exactly the same implementation as the one employed in real end-terminal prototypes. Likewise, we have emulated the adaptive applications, the end-terminal context changes (e.g., battery state changes) and the "Lower-Layers" (i.e., networks QoS parameters changes) as shown in Fig. 5. Thus we were able to study multi-access middleware (i.e., Profile Manager, Selection Decision modules and Profiles Database) within our emulator playing realistic scenarios.

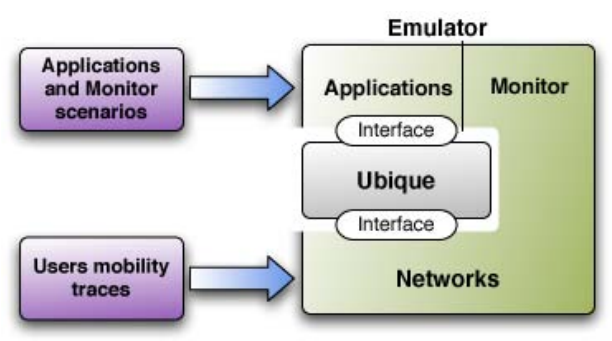

Figure 5: Emulator architecture and interactions

In order to evaluate the advantages of PRP management integration in a vertical handoff mechanism we compare Ubique and a limited version of Ubique that do not take into account PRPs. We model the battery state changes and the arrival/departure of applications (composite traffic flows) as a Poisson Process with a mean arrival interval $(1 / \lambda)$ of $1 \mathrm{~h}$ for battery state and $15 \mathrm{~min}$ for applications, and mean service time $(1 / \mu)$ of $15 \mathrm{~min}$ for applications. Finally, the power consumption and the parameters we used for the network technologies (NIP) come from [15].

\subsection{Results and discussions}

All the results introduced in this section are obtained from the simulation scenario presented in the previous paragraph. We considered 20 mobile terminals (i.e., the technicians). The simulation time (i.e., the working day) is set to $8 \mathrm{~h}$. Finally, the simulation area is $6 \mathrm{~km}$ (a town).

\subsubsection{PRP impact on costs}

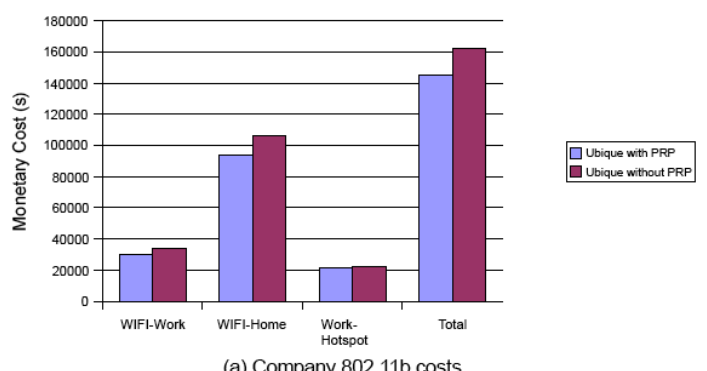

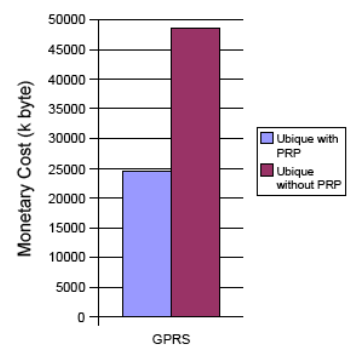

(b) Company GPRS costs

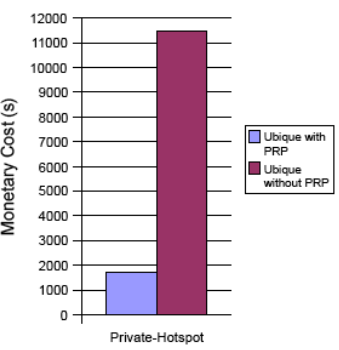

(c) Users costs

Figure 6: Monetary costs 
Figs. 6 (a) and 6 (b) show the cost induced for the company which is the sum of cost generated by all technicians. It can be immediately noticed the interest of PRP management to control the fleet monetary costs. Results show a clear reduction of the overall cost when Ubique is used with PRP. For example, in Fig. 6 (b), we see that the GPRS monetary cost under Ubique is nearly $50 \%$ of the one that is obtained using legacy vertical handover mechanism.

This behavior is due to the fact that Ubique takes into account the purposes of the application (professional or leisure), therefore the overall cost is more controlled. In fact, in our scenario as it is shown in Table 3, the fleet administrator specified on the one hand that employees can not launch their personal applications such as I-Chat and VoIP through a professional subscription charged to the company (i.e., GPRS, WIFI-Work, WIFI-Home and Work-Hotspot ). Thus, Ubique will not take into account these expensive access networks when selecting the best interface for personal applications.

On the other hand, the administrator declared GPRS and Work-Hotspot as forbidden for some professional applications which are not considered as urgent such as E-mail and SSH. In Fig. 6 (c) we can see user's monetary costs.

The large difference in monetary costs between the two vertical handoff mechanisms is due to the PRP mechanism which enables users to use the paying network access (i.e. Private-Hotspot) only for critical applications sensitive to delay (i.e. Visio-Conf-Perso and VoIP-Perso). We note from this results that the PRP management allows the vertical handoff management mechanism to select the best interfaces taking into account the purposes of the applications, their needs and the context in which the terminal operates.

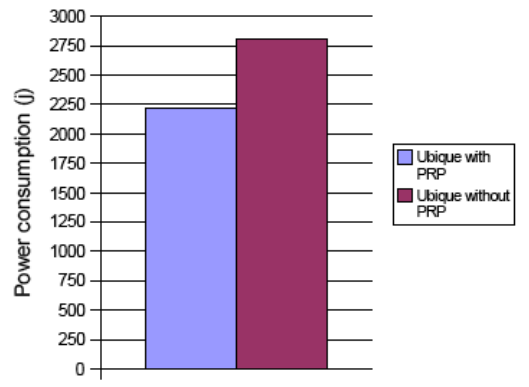

Figure 7: Fleet power consumption

Fig. 7 shows the power consumption due to communications. We can see that the PRP management module allows the administrator to reduce the power consumption of the fleet. This is due to the fact that PRP lets vertical handoff mechanism take into account the power consumption in the handover decision process. For example, in our scenario, as it is shown in Tables 3 and 4, when the battery state is "Low", on the one hand GPRS is declared "Forbidden network" for all applications as this technology consumes more energy than IEEE802.11b and Bluetooth. On the other hand, the administrator specified in PRP that not critical professional applications can be launched only on the less consuming interface (i.e., the Bluetooth interface). Moreover, the Administrator prohibits users to employ their personal applications when the battery is "low" reserving re- maining energy for professional purposes.

\subsubsection{PRP impact on applications satisfaction}

Results presented in the previous section show that the PRP management mechanism integrated in our vertical handoff decision middleware allows a fine control over the handover decision made locally in mobile node. It allows administrator of the fleet to reduce the overall communication cost and the energy consumption which could be crucial depending on the activity. Moreover, simple XML configuration files downloaded in the terminal are enough to achieve this result.
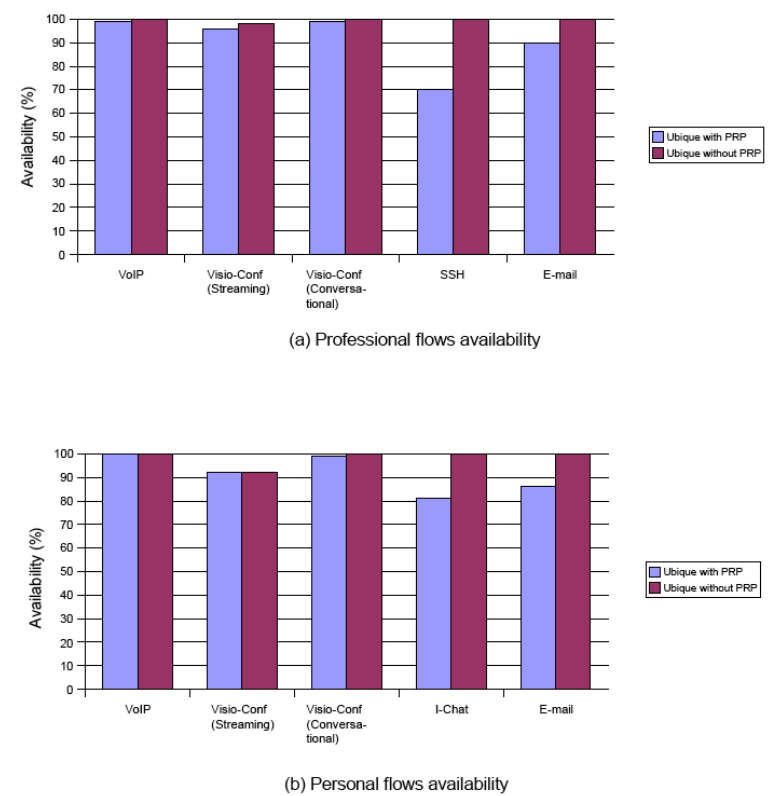

Figure 8: Professional flows availability

Nonetheless, there are several challenging issues we still have to check about the PRP integration. Is it able to improve or at least not to reduce flow satisfaction especially when flows have strong QoS requirement? Some of them may even be critical for the technician activity.

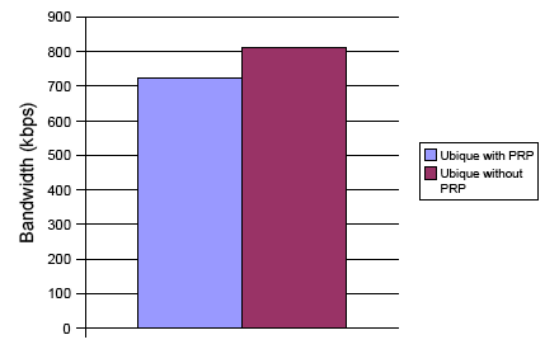

Figure 9: Average bandwidth by flow

Fig. 8 shows flows availability rate with and without PRP. These figures show that critical applications flows such as VoIP and Visio-Conference flows have approximatively the same amount of availability whatever PRP is used or not. Non-critical applications flows such as I-Chat, E-mail-Perso 
and E-mail-Work have slightly more availability rate when the PRP management mechanism is not used. This is due to the fact that PRP usage prevent them to use ressources they are not allowed to use. It is important to note that the PRP mechanism does not have an important effect on critical applications availability, thus on employees productivity; it has only impact on the non-critical applications.

In Fig. 9 we can see the average bandwidth allocated to each traffic flow during the simulation. We notice that application flows receive slightly less bandwidth when the PRP management module is activated. Nonetheless, it can be pointed out that the flow bandwidth remain largely sufficient to launch any application; indeed, the maximum flow bandwidth required is $64 \mathrm{kbps}$ (see Table 2). This way no useless bandwidth is allocated.

\section{CONCLUSIONS AND FUTURE WORK}

In this paper we have presented an adaptable and reconfigurable middleware for the mobile terminals supporting multiple network interfaces. Our first ambition was to provide users with seamless access over heterogeneous networks. We also aim at providing an easy way to manage multiple level of preferences.

This middleware allows the management of multiple-interfaces mobile terminals fleets and control their monetary cost and energy consumption. The proposed middleware includes adaptation mechanisms and relies on profiles handling to feed the vertical handover decision algorithm.

On-going works focus on further refinement of profiles, e.g., uniform monetary cost representation. In addition, more fleets management strategies need to be investigated and the most promising of them will be implemented and evaluated.

\section{REFERENCES}

[1] 3GPP TS 23.234, "3GPP system to Wireless Local Area Network (WLAN) Interworking", System Description (Release 6) V2.3.0, November 2003

[2] F. Siddiqui and S. Zeadally, "Mobility management across hybrid wireless networks: Trends and challenges", In Computer Communications, (29):1363-1385, 2006.

[3] F. André and al., "Optimised support of multiple wireless interfaces within an IPv6 end-terminal", In Smart Objects Conference., May 2003

[4] F. Fitzek and al., "Providing Application-Level QoS in 3G/4G Wireless Systems: A Comprehensive Framework Based on Multirate CDMA", In IEEE Wireless Communications, April 2002

[5] T. Eskedal and al., "Inter-Operator IP QoS Framework - ToIP and UMTS Case Studies", EURESCOM Project Report P1103, January 2002

[6] E. Gustafsson and A. Jonsson, "Always best connected", In IEEE Wireless Communications, February 2003

[7] M. O'Droma and al., "Always best connected enabled 4G wireless world", In IST Mobile and Wireless Communications Summit 2003 June 2003

[8] T. Park and A. Dadej, "Adaptive handover control in IP-based mobility networks", In Proc. of 1st Workshop on the Internet, Telecommunications and Signal Processing (WITSP'02), December 2002
[9] E. Stevens-Navarro and V.W.S. Wong, "Comparison between Vertical Handoff Decision Algorithms for Heterogeneous Wireless Networks", In IEEE Vehicular Technology Conference (VTC), Melbourne, Australia, May 2006

[10] T. Zahariadis, "Trends in the path to $4 \mathrm{G}$. Communications Engineer", In IET Communications Engineer February 2003

[11] 3GPP TS 24.241 V0.4.0, "3GPP generic user profile (GUP) common objects", Stage 3. 3GPP, August 2003

[12] WAP 248-UAPROF V20, "User agent profiling specification", WAP Forum, October 2001

[13] W3C, "Composite Capability/Preference Profiles (CC/PP): Structure and Vocabularies 1.0", W3C Draft, January 2004

[14] M. Williams, "Directions in Media Independent Handover", In IEICE Transactions on Fundamentals of Electronics, Communications and Computer Sciences - Special Section on Multi-dimensional Mobile Information Networks, July 2005

[15] B. Xing and N. Venkatasubramanian, "Multi-Constraint Dynamic Access Selection in Always Best Connected Networks", In IEEE MobiQuitous'05, pp. 56-64, San Diego, CA, July 2005

[16] Y. Collette and P. Siarry, "Multiobjective Optimization - Principles and Case Studies", Springer, August 2003

[17] W. Zhang, J. Jaehnert and K. Dolzer "Design and evaluation of a handover decision strategy for 4th generation mobile networks", In 57th Semiannual Vehicular Technology Conference VTC 2003-Spring, April 2003

[18] Kibria, M. R. and Jamalipour, A. and Mirchandani, V., "A location aware three-step vertical handoff scheme for 4G/B3G networks", In IEEE Global Telecommunications Conference, 2005, Kibria, November 2005

[19] Fang Zhu and Janise McNair, "Multiservice Vertical Handoff Decision Algorithms", In EURASIP Journal on Wireless Communications and Networking 2006

[20] Quoc-Thinh Nguyen-Vuong and Nazim Agoulmine and Yacine Ghamri-Doudane, "Terminal-Controlled Mobility Management in Heterogeneous Wireless Networks", In IEEE_M_COM, Nguyen-Vuong2007, April 2007

[21] Ling-Jyh Chen, Tony Sun, Benjamin Cheung, Duke Nguyen, and Mario Gerla, "Universal Seamless Handoff Architecture in Wireless Overlay Networks", Technical Report TR040012, UCLA CSD 2004

[22] S.K. Lee, K. Sriram, K. Kim, J.H. Lee, Y.H. Kim, and N. Golmie, "Vertical Handoff Decision Algorithms for Providing Optimized Performance in Heterogeneous Wireless Networks", In IEEE GLOBECOM 2007, Washington D.C, November 2007

[23] M. Mahamat-faki, J.-M. Bonnin, and R. Ben Rayana, "Traffic flow control on multi-tunneled mobile router", In WNEPTO6 (Workshop on Networking in Public Transport), Waterloo, Canada, August 2006.

[24] L. Suciu and J.-M. Bonnin, "A survey of multi-criteria network selection algorithms", In Global Mobile Congress (GMC'04), Shanghai, Chine, October 2004. 